\title{
A metabolic milieu
}

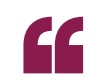

\section{there is a \\ growing \\ appreciation \\ for CF lungs}

as hosts to a

polymicrobial

community
Cystic fibrosis (CF) is a complex disease, and our understanding of the resulting physiological alterations is still incomplete. One well-studied consequence of CF is the build-up of abnormally thick mucus in the lungs, which is home to a range of microorganisms. Infections in the CF lung are often thought of purely in terms of Pseudomonas aeuruginosa; however, although this opportunistic pathogen is important, there is a growing appreciation for CF lungs as hosts to a polymicrobial community. Reporting in $\mathrm{mBio}$, Quinn et al. now examine the core metabolic functions within the CF lung microbiome.

Microbial DNA and RNA were extracted from sputum samples that were taken from six different patients with $\mathrm{CF}$, and high-throughput sequencing was used to obtain metagenomic and metatranscriptomic data. The sequence reads that were generated were assigned to functional categories by matching them to the KEGG

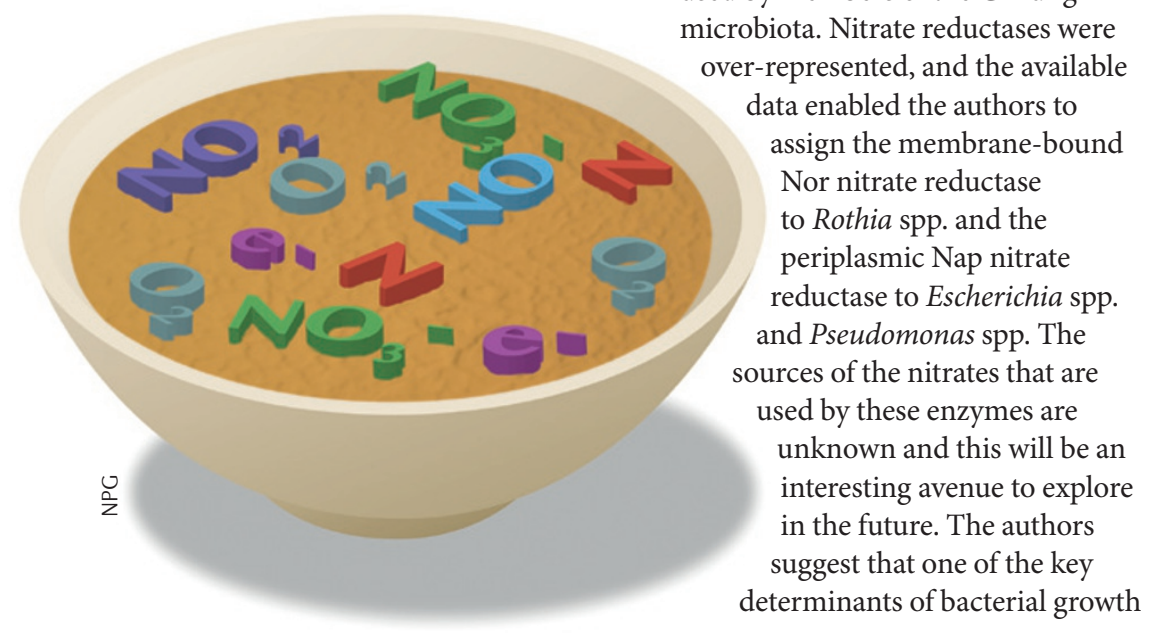

(Kyoto Encyclopedia of Genes and Genomes) database.

To assess the prevalent types of microbial metabolism in the CF lung, the authors identified metabolic functions that were enriched in the CF lung microbiome compared with the microbiome present in saliva samples taken from healthy individuals. The abundant pathways included those involved in amino acid metabolism and oxidative phosphorylation. Fermentation pathways were also enriched; this suggests that differential oxygen gradients in the CF lung may require alternative respiratory strategies. Interestingly, folate biosynthesis pathways were also abundant in the CF lung microbiome, which the authors suggest could reflect the widespread use of sulphonamides to treat infections in patients with CF.

The authors went on to take a closer look at energy metabolism. The genomic and transcriptomic data indicated that a range of primary electron acceptors can be used by members of the CF lung microbiota. Nitrate reductases were data enabled the authors to assign the membrane-bound Nor nitrate reductase to Rothia spp. and the periplasmic Nap nitrate reductase to Escherichia spp. and Pseudomonas spp. The sources of the nitrates that are used by these enzymes are unknown and this will be an interesting avenue to explore in the future. The authors determinants of bacterial growth rate within the CF lung could be the availability of alternative electron acceptors, and so one reason for the success of $P$. aeruginosa might be its ability to express redox-active phenazines, which can shuttle electrons to external donors. The same group also recently observed a $\mathrm{pH}$-neutral fermentation product, 2,3-butanedione, in CF breath samples, which may increase phenazine production. Finally, an in-depth characterization of the nitrogen cycle revealed the abundance of genes encoding proteins that are involved in denitrification but the absence of genes encoding proteins that are involved in the corresponding oxidative reactions. The absence of a complete nitrogen cycle, together with the abundance of pathways for amino acid catabolism, suggests that ammonia might accumulate in the CF lung, and this was confirmed by the direct measurement of nitrogen species in a CF bronchiole culture model.

This analysis of the biochemical signatures that are associated with the CF lung microbiome should facilitate our understanding of the host-microbiome interactions that occur within the CF lung and may also provide valuable leads for the development of new therapeutic agents.

Sheilagh Molloy

ORIGINAL RESEARCH PAPER Quinn, R. A. et al. Biogeochemical forces shape the composition and physiology of polymicrobial communities in the cystic fibrosis lung. mBio 5, e00956-13 (2014). FURTHER READING Whiteson, K. et al. Breath gas metabolites and bacterial metagenomes from cystic fibrosis airways indicate active $\mathrm{pH}$ neutral 2,3-butanedione fermentation. ISMEJ. http:// dx.doi.org/10.1038/ISMEJ.2013.229 (2014) 